\title{
Interfaces in the Potts Model I: Pirogov-Sinai Theory of the Fortuin-Kasteleyn Representation
}

\author{
Lahoussine Laanait^, Alain Messager, Salvador Miracle-Sole, Jean Ruiz \\ and Senya Shlosman $\star \star$
}

Centre de Physique Théorique ${ }^{\star \star \star}$, CNRS-Luminy-Case 907, F-13288 Marseille Cedex 9, France

Received August 1, 1990

\begin{abstract}
We develop a new analysis of the order-disorder transition in ferromagnetic Potts models for large number $q$ of spin states. We use the Pirogov-Sinaï theory which we adapt to the Fortuin-Kasteleyn representation of the models. This theory applies in a rather direct way in our approach and leads to a system of non-interacting contours with small activities. As a consequence, simpler and more natural techniques are found, allowing us to recover previous results on the bulk properties of the model (which then extend to non-integer values of $q$ ) and to deal with non-translation invariant boundary conditions. This will be applied in a second part of this work to study the behaviour of the interfaces at the transition point.
\end{abstract}

\section{Introduction}

When we consider three phases, $a, b$ and $c$, in thermal equilibrium, the situation may arise in which a layer of the phase $c$ is developed at the boundary between the $a$ and $b$ phases in order to lower the surface tension (i.e. the free energy). Then two interfaces appear: one between $a$ and $c$, the other between $c$ and $b$. The interaction between these two interfaces leads to a large variety of physical phenomena.

A theoretical example of this situation is provided by the ferromagnetic $q$-state Potts model in dimension $d \geqq 2$. This model exhibits $q$ ordered phases at low temperature and one disordered phase at high temperature. When the orderdisorder transition is first order, which is the case when $q$ is large enough, all phases

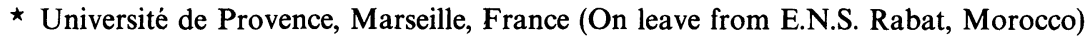

$\star \star$ On leave from Institute for Problems of Information Transmission of the Academy of Sciences, Moscow, USSR

$\star \star \star$ Laboratoire Propre du CNRS: LP 7061
} 
remain distinct at the transition temperature and the situation described above appears: $a, b$, and $c$ being respectively two ordered and the disordered phase.

Our purpose here is to develop appropriate mathematical techniques for analyzing the behaviour of the Potts model at the transition point. The main result will be the description of the model as a gas of polymers (contours) and from it the derivation of a cluster or "low temperature" expansion in powers of $q^{-1 / d}$ which converges for $q$ large enough. An application of these techniques to the problem of three coexisting phases will be discussed in a forthcoming paper.

In the Potts model [1] spin variables $\sigma_{i}$ which take values on a discrete set $\{1,2, \ldots, q\}$ are associated with each site $i$ of the lattice. Two adjacent spins $\sigma_{i}$ and $\sigma_{j}$ interact with interaction energy $-J \delta\left(\sigma_{i}, \sigma_{j}\right)$, where $J>0$ for ferromagnetic systems and $\delta$ is the Kroeneker symbol. Therefore, the total energy is $H=-J \sum \delta\left(\sigma_{i}, \sigma_{j}\right)$ and the partition function is

$$
Z(V)=\sum_{\sigma} \exp \left\{\beta \sum_{\langle i, j\rangle} \delta\left(\sigma_{i}, \sigma_{j}\right)\right\},
$$

where the sum runs over all configurations inside a box $V$ and $\beta=J / k T$. A first order phase transition when $\beta$ varies is expected for $q \geqq 5$ in $d=2$ and for $q \geqq 3$ in $d=3$ [2]. This has been proved from the exact solution in the case $d=2$ [3] and, provided that $q$ is large enough, in any dimension $d \geqq 2$ [4-8].

It was shown by Fortuin and Kasteleyn [9] that the partition function $Z$ is equal to

$$
Z(V)=\sum_{X}\left(e^{\beta}-1\right)^{|X|} q^{N_{V}(X)},
$$

where the summation is over all graphs $X$ (subsets of bonds) which can be drawn inside the box $V$, containing $|X|$ bonds and $N_{V}(X)$ connected components (regarding an isolated point as a component). Note that $q$ need not be an integer, we can allow it to be any positive number.

Our approach is based on the theory of Pirogov and Sinaï $[10,11]$ which we adapt to the Fortuin-Kasteleyn representation of the model. With respect to precedent works dealing also with the large $q$ case [4-8], let us mention that the Pirogov and Sinaï results apply in this approach in a rather direct way, without introducing new concepts or generalizations, and lead to a system of non-interacting contours. The following observations explain the reason why these results apply to the considered system.

Let us consider the $d$-dimensional cubic lattice $\mathbb{Z}^{d}$ and denote by $\mathscr{B}$ the set of bonds (pairs of nearest neighbours), the subsets $X \subset \mathscr{B}$ of occupied bonds describe then the configurations of the system. One says that two configurations $X$ and $Y$ are equal almost everywhere $(X=Y$ a.e.) if they differ only a finite set of bonds, i.e. if there exist a finite $V \subset \mathscr{B}$ such that $X \cap V^{c}=Y \cap V^{c}$, where $V^{c}=\mathscr{B} \backslash V$. We denote by $S(X)$ the set of sites which belong to some bond in $X$ and by $C(X)$ the number of connected subsets, single sites are not included. Given two configurations $X, Y$ such that $X=Y$ a.e., we define their relative energy by

$$
H(X \mid Y)=-\ln \left(e^{\beta}-1\right)(|X|-|Y|)-\ln q(-|S(X)|+|S(Y)|+C(X)-C(Y)),
$$

according to the Boltzmann weight which appears in the Fortuin-Kasteleyn representation. Hereafter $|E|$ denotes the cardinality of the set $E$. 
A configuration $Y$ is a ground configuration if $H(X \mid Y) \geqq 0$ for all $X$ such that $X=Y$ a.e. It is not difficult to see that the empty set $\varnothing$ and the full set $\mathscr{B}$, which we shall also call (following [12]) the free and the wired configurations, are ground configurations for some values of $\beta$ and $q$. In fact, $X=\varnothing$ a.e. and $X=\mathscr{B}$ a.e. respectively mean that $X$ and $X^{c}$ are finite sets. In the first case we have

$$
H(X \mid \varnothing)=-\ln \left(e^{\beta}-1\right)|X|-\ln q(-|S(X)|+C(X)),
$$

and in the second case

$$
H(X \mid \mathscr{B})=\ln \left(e^{\beta}-1\right)\left|X^{c}\right|-\ln q\left(\left|S_{I}\left(X^{c}\right)\right|+C(X)-1\right),
$$

where $S_{I}(X)$ is the subset of $S(X)$ such that any element is the endpoint of $2 d$ bonds of $X$. If $\ln \left(e^{\beta}-1\right) \leqq(1 / d) \ln q$ and $X=\varnothing$ a.e., then

$$
H(X \mid \varnothing) \geqq \ln q(|S(X)|-(1 / d)|X|-C(X)) \geqq 0,
$$

and if $\ln \left(e^{\beta}-1\right) \geqq(1 / d) \ln q$ and $X=\mathscr{B}$ a.e., then

$$
\begin{aligned}
H(X \mid \mathscr{B}) & \geqq \ln q\left(-\left|S_{I}\left(X^{c}\right)\right|+(1 / d)\left|X^{c}\right|-C(X)+1\right) \\
& =\ln q(|S(X)|-(1 / d)|X|-C(X)+1) \geqq 0 .
\end{aligned}
$$

We note that in order to make the difference $|S(X)|-(1 / d)|X|$ in (1.4) well defined, one has to go from the lattice $\mathbb{Z}^{d}$ to the discrete torus $\mathbb{T}_{N}^{d}$ of size $N$ (then $|S(X)|-(1 / d)|X|$ does not depend on $N$ for $N$ large). The positivity of the last two expressions (1.3) and (1.4) is proven by easy geometrical arguments (see the Lemma of Sect. 2 and use the remark that each finite connected subset of $X$ must be surrounded by more than $2 d$ elements of $\left.X^{c}\right)$. This says that the free and wired configurations are ground configurations if $\beta \leqq \ln \left(q^{1 / d}+1\right)$ and $\beta \geqq \ln \left(q^{1 / d}+1\right)$ respectively. The same arguments show that both expressions have a uniform lower bound proportional to the number of elements of the boundary of $X$. (Since the set $S(X) \cap S\left(X^{c}\right)$ contains the sites where the configuration $X$ looks different from the two ground configurations we may take it as a definition of the boundary.) This means that the Peierls condition needed in the Pirogov-Sinaï approach is satisfied.

The precise statements which follow from the approach described above are discussed in Sect. 2. In Sect. 3 some consequences of these results are explained.

\section{Main Results}

2.1. Notations and Definitions. Two bonds are adjacent if they have an endpoint in common. Two bonds are co-adjacent if a $d$-cell exists containing these two bonds ( $d$-cells are plaquettes for $d=2$, cubes for $d=3$, etc.).

A set of bonds, $X$, is connected (respectively co-connected) if for any $b$ and $b^{\prime}$ in $X$, there is a sequence of bonds in $X, b=b_{1}, b_{2}, \ldots, b_{n}=b^{\prime}$ such that $b_{i}$ and $b_{i+1}$ are adjacent (respectively co-adjacent) for all $i=1, \ldots, n-1$. We note that to any $p$-cell in $\mathbb{Z}^{d}$ we can associate the orthogonal $(d-p)$-cell of the dual lattice $(\mathbb{Z}+1 / 2)^{d}$ which intersects it. The co-connectedness of a set of bonds means that its dual is a connected set of $\mathbb{R}^{d}$ (with the usual topology). 
For any set of bonds $X \subset \mathscr{B}$, we define the boundary $\partial X$ as the set of bonds which belong to $X$ and are co-connected to the complementary $X^{c}=\mathscr{B} \backslash X$. We define the co-boundary $\delta X$ as the set of bonds which belong to $X^{c}$ and are connected to $X$. Then, $\delta X=\delta_{1} X \cup \delta_{2} X$, where

$$
\begin{aligned}
& \delta_{1} X=\left\{b \in X^{c} /|S(b) \cap S(X)|=1\right\}, \\
& \delta_{2} X=\left\{b \in X^{c} /|S(b) \cap S(X)|=2\right\} .
\end{aligned}
$$

and the following geometrical property holds

\section{Lemma.}

$$
|S(X)|-\frac{1}{d}|X|=\frac{1}{2 d}\left[\left|\delta_{1} X\right|+2\left|\delta_{2} X\right|\right]
$$

The proof follows from the corresponding definitions and the fact that $2 d$ bonds meet at every point of the lattice.

We introduce the partition functions with free and wired boundary conditions:

$$
\begin{aligned}
& Z^{f}(V)=\sum_{X \subset V ; X \cap \delta V c=\varnothing}\left(e^{\beta}-1\right)^{|X|} q^{N_{V}}(X), \\
& Z^{w}(V)=\sum_{X \subset V ; X \supset \partial V}\left(e^{\beta}-1\right)^{|X|} q^{N_{V}}(X),
\end{aligned}
$$

where $X \subset \mathscr{B}$ and $N_{V}(X)=C(X)+\left|S_{I}(V) \backslash S(X)\right|$. The following limits (independent of the boundary conditions)

$$
f(\beta)=\lim _{V \uparrow \mathscr{B}}(1 /|V|) \ln Z^{f}(V)=\lim _{V \uparrow \mathscr{B}}(1 /|V|) \ln Z^{w}(V)
$$

exist and $-(1 / \beta) f(\beta)$ gives the free energy per bond.

2.2. Contours. The co-boundary of a set of bonds split into co-connected components which we shall call contours. Our aim is to express the partition functions $Z^{w}$ and $Z^{f}$ in terms of two contour models associated with the functionals $\phi_{w}$ and $\phi_{f}$ defined respectively on the set of $w$-contours and on the set of $f$-contours.

A co-connected subset $\gamma$ of $\mathscr{B}$ is called a contour if it is the co-boundary of some set $X \subset \mathscr{B}$, such that either $X$ or $X^{c}$ is a finite set. In the first case we say that $\gamma$ is a contour of the free class, or a $f$-contour, in the second case we say that $\gamma$ is a contour of the wired class, or a w-contour. The unique infinite component of $\mathscr{B} \backslash \gamma$ is denoted by Ext $\gamma$ and we define also the sets $V(\gamma)=\mathscr{B} \backslash$ Ext $\gamma$, and Int $\gamma=V(\gamma) \backslash \gamma$ and the length of the contour

$$
\begin{array}{ll}
\|\gamma\|=\left|\delta_{1} \operatorname{Int} \gamma\right|+2\left|\delta_{2} \operatorname{Int} \gamma\right|, & \text { if } \gamma \text { is a } f \text {-contour, } \\
\|\gamma\|=\left|\delta_{1} \operatorname{Ext} \gamma\right|+2\left|\delta_{2} \operatorname{Ext} \gamma\right|, & \text { if } \gamma \text { is a } w \text {-contour. }
\end{array}
$$

Two non-intersecting contours $\gamma_{1}, \gamma_{2}$ are called mutually compatible. Two contours of the same class are called mutually compatible external contours if $V\left(\gamma_{1}\right) \subset \operatorname{Ext} \gamma_{2}$ and $V\left(\gamma_{2}\right) \subset \operatorname{Ext} \gamma_{1}$. If $\theta=\left\{\gamma_{1}, \gamma_{2}, \ldots, \gamma_{n}\right\}$ is a family of mutually compatible external contours we denote: $V(\theta)=\bigcup V\left(\gamma_{i}\right)$, Int $\theta=V(\theta) \backslash \theta$, Ext $\theta=$ $\mathscr{B} \backslash V(\theta)$, and $\operatorname{Ext}_{V} \theta=V \cap \operatorname{Ext} \theta$.

Let $X \subset \mathscr{B}$ and assume that $X^{c}$ is a finite set. Denote by $X_{\infty}$ the unique infinite connected component of $X$. Then the co-boundary $\delta X_{\infty}$ of $X_{\infty}$ split into 
co-connected components $\gamma_{1}, \ldots, \gamma_{n}$, which are mutually compatible external contours of the wired class and will be called the external contours of $X$.

Let $X \subset \mathscr{B}$ and assume that $X$ is a finite set. Denote by $X_{\infty}^{c}$ the unique infinite co-connected component of $X^{c}$. Then the co-boundary of $\mathscr{B} \backslash X_{\infty}^{c}$ split into co-connected components $\gamma_{1}, \ldots, \gamma_{n}$, which are mutually compatible external contours of the free class and will be called the external contours of $X$.

With these definitions we obtain the following inductive expressions for the partition functions:

$$
Z^{w}(V)=\sum_{\theta_{w} \subset V} q^{C(V)}\left(e^{\beta}-1\right)^{\left|\operatorname{Ext}_{V} \theta_{w}\right|} Z^{f}\left(V\left(\theta_{w}\right)\right),
$$

where the sum runs over the families $\theta_{w}$ of mutually compatible external $w$-contours $\gamma$ included in $V$ such that $\gamma \cap \partial V=\varnothing$, which we hereafter denote by $\theta_{w} \subset V$, and

$$
Z^{f}(V)=\sum_{\theta_{f} \subset V} q^{\left|S_{I}\left(V \backslash \operatorname{Int} \theta_{f}\right)\right|} Z^{w}\left(\operatorname{Int} \theta_{f}\right),
$$

where the sum runs over the families $\theta_{f}$ of mutually compatible external $f$-contours ix:cluded in $V$.

2.3. Contour Models. The partition function, in a volume $V(V \subset \mathscr{B})$, of a contour model $\phi$ with contour weights $\phi(\gamma)$ is given by

$$
\mathscr{Z}(V \mid \phi)=\sum_{\partial \subset V} \prod_{\gamma \in \partial} \phi(\gamma)
$$

where the sum is over all admissible families $\partial$ of contours in $V$ : this will mean that the $\gamma_{w}$ 's are included in $V$ and such that $\gamma_{w} \cap \partial V=\varnothing$ for families $\partial_{w}$ of compatible $w$-contours and that the $\gamma_{f}$ 's are included in $V$ for families $\partial_{f}$ of compatible $f$-contours.

The partition function of a contour model $\phi$ with a parameter $b$ is defined by

$$
\mathscr{Z}(V \mid \phi, b)=\sum_{\theta \subset V} \prod_{\gamma \in \theta} e^{b|V(\gamma)|} \phi(\gamma) \mathscr{Z}(\operatorname{Int} \gamma \mid \phi),
$$

where the sum runs over families $\theta$ of mutually external contours.

To have a good control on partition functions of contour models one relies on their " $\tau$-functionality"; $\phi$ is called a $\tau$-functional if for some fixed number $\tau>0$ and every $\gamma$, it satisfies the estimates

$$
|\phi(\gamma)| \leqq e^{-\tau\|\gamma\|} .
$$

This ensures, in particular, the existence of the limit

$$
f(\phi)=\lim _{V \uparrow \mathscr{B}|V|} \frac{1}{\mid n} \ln (V \mid \phi),
$$

which is the free energy per bond. We may now state the main result of the present work.

Theorem. Assume that $q$ is large enough and $d \geqq 2$. Then for every $\beta$ there exist non-negative parameters $b_{w}, b_{f}$ and associated $\tau$-functionals $\phi_{w}^{b_{w}}, \phi_{f}^{b_{f}}$ such that:

$$
b_{w}+\ln \left(e^{\beta}-1\right)+f\left(\phi_{w}^{b_{w}}\right)=b_{f}+\frac{1}{d} \ln q+f\left(\phi_{f}^{b_{f}}\right)=f(\beta),
$$




$$
\begin{gathered}
Z^{w}(V)=q^{C(V)}\left(e^{\beta}-1\right)^{|V|} \mathscr{Z}\left(V \mid \phi_{w}^{b_{w}} ; b_{w}\right), \\
Z^{f}(V)=q^{S_{I}(V)} \mathscr{Z}\left(V \mid \phi_{f}^{b_{f}} ; b_{f}\right) .
\end{gathered}
$$

There exists a unique inverse temperature $\beta_{t}=\beta_{t}(q, d)$ such that

$$
\begin{array}{llll}
b_{w}=0 & \text { and } & b_{f}=0 & \text { for } \beta=\beta_{t}, \\
b_{w}=0 & \text { and } & b_{f}>0 & \text { for } \beta>\beta_{t}, \\
b_{w}>0 & \text { and } & b_{f}=0 & \text { for } \beta<\beta_{t} .
\end{array}
$$

The contour functionals $\phi_{w}^{b_{w}}$ and $\phi_{f}^{b_{f}}$ are defined inductively by:

$$
\begin{aligned}
\phi_{w}^{b_{w}}\left(\gamma_{w}\right) \mathscr{Z}\left(\operatorname{Int} \gamma_{w} \mid \phi_{w}^{b_{w}}\right) & =e^{-b_{w}\left|V\left(\gamma_{w}\right)\right|}\left(e^{\beta}-1\right)^{-\left|V\left(\gamma_{w}\right)\right|} Z^{f}\left(V\left(\gamma_{w}\right)\right), \\
\phi_{f}^{b_{f}}\left(\gamma_{f}\right) \mathscr{Z}\left(\operatorname{Int} \gamma_{f} \mid \phi_{f}^{b_{f}}\right) & =e^{-b_{f}\left|V\left(\gamma_{f}\right)\right|} q^{\left.-\mid \operatorname{S(Int} \gamma_{f}\right) \mid} Z^{w}\left(\operatorname{Int} \gamma_{f}\right),
\end{aligned}
$$

and satisfy the estimates:

$$
\begin{aligned}
& \phi_{w}^{b_{w}}\left(\gamma_{w}\right) \leqq q^{-(1 / 2 d)\left\|\gamma_{w}\right\|} \exp \left\{9 q^{-1 / 2 d}\left\|\gamma_{w}\right\|\right\}, \\
& \phi_{f}^{b_{f}}\left(\gamma_{f}\right) \leqq q^{-(1 / 2 d)\left\|\gamma_{f}\right\|+C\left(\operatorname{lnt} \gamma_{f}\right)} \exp \left\{6 q^{-[(d-1) /(4 d-2) d]}\left\|\gamma_{f}\right\|\right\} .
\end{aligned}
$$

The proof of the theorem is given in the Appendix. Let us notice that the inductive expression (2.15) of contour functionals immediately yields relations (2.12) and (2.13) of the theorem, taking into account the inductive expressions $(2.5)$ and (2.6) of $Z^{w}$ and $Z^{f}$ and the definition (2.8) of the partition function $\mathscr{Z}(V \mid \phi, b)$ of a contour model with a parameter. The statement (2.11) also follows from (2.15) by taking the logarithms, provided $\phi_{w}^{b_{w}}$ and $\phi_{f}^{b_{f}}$ are indeed contour functionals. Because $S(X) /|X| \rightarrow 1 / d$ when $X \uparrow \mathscr{B}$ we have the term $(1 / d) \ln q$ in (2.11).

We get then, the following expression for the transition temperature

$$
\ln \left(e^{\beta_{t}}-1\right)=(1 / d) \ln q+f\left(\phi_{f}^{0}\right)-f\left(\phi_{w}^{0}\right),
$$

where the free energies $f\left(\phi_{w}^{0}\right)$ and $f\left(\phi_{f}^{0}\right)$ are given for large $q$ by

$$
\begin{aligned}
& f\left(\phi_{w}^{0}\right)=q^{-1 / d}+O\left(q^{-2 / d}\right), \\
& f\left(\phi_{f}^{0}\right)=q^{-(d-1) / d}+O\left(q^{-2(d-1) / d}\right) .
\end{aligned}
$$

Indeed this may be seen from the convergent contours expansions of the theorem, taking into account the fact that the first terms in the expansions come from the smallest contours. In the wired case the smallest contour has length 2 and the other ones have length at least 4 . In the free case the smallest contour has length $4 d-2$ with one component for its interior and the other ones have length at least $6 d-4$.

Notice that for $d=2$, the duality relation (which in the considered representation corresponds to the exact symmetry of the model $[3,13]$ under the transformation $X \rightarrow V \backslash X)$ implies $f\left(\beta^{*}\right)=\ln \left[\left(e^{\beta}-1\right) q^{-1 / 2}\right]+f(\beta)$, where $\beta^{*}$ is such that $\ln \left[\left(e^{\beta^{*}}-1\right) q^{-1 / 2}\right]=-\ln \left[\left(e^{\beta}-1\right) q^{-1 / 2}\right]$. From the theorem the transition point $\beta_{t}$ is unique, therefore $\beta_{t}^{*}=\beta_{t}$ if $d=2$, which gives

In dimensional $d \geqq 3$ we have

$$
\ln \left(e^{\beta_{t}}-1\right)=(1 / 2) \ln q \text {. }
$$

$$
\ln \left(e^{\beta_{t}}-1\right)=(1 / d) \ln q+q^{-1 / d}+O\left(q^{-2 / d}\right),
$$


as a consequence of (2.17) and (2.18).

The theorem above shows, as was mentioned in the Introduction, that the Fortuin-Kasteleyn version of the Potts model may equivalently be described as a system of non-interacting compatible contours. If $q$ is large enough the associated activities are small and decay exponentially in the contour length. This allows a good control on the behaviour of the system at any temperature.

\section{Concluding Remarks}

The expressions in terms of contours of the partition functions under free and wired boundary conditions are given by the theorem above. We introduce also the Gibbs states $\langle\cdot\rangle^{w}$ and $\langle\cdot\rangle^{f}$ associated to these boundary conditions. For a fixed bond $b$ of the lattice, let $n_{b}$ be the occupation number: $n_{b}(X)=1$ if $b$ belongs to $X$ and 0 otherwise. For $\beta=\beta_{t}$ and if $q$ is large enough we have $\left\langle n_{b}\right\rangle^{f} \leqq O\left(q^{-(d-1) / d}\right)$, because a bond is occupied only if there is a contour $\gamma$ surrounding it, $\left|\phi_{f}^{0}(\gamma)\right|$ is an upper bound on the probability of the contour and the shortest $f$-contour has length $\|\gamma\|=2(2 d-1)$. Similarly $\left\langle 1-n_{b}\right\rangle^{w} \leqq O\left(q^{-1 / d}\right)$ because the shortest $w$-contour has length $\|\gamma\|=2$, therefore $\left\langle n_{b}\right\rangle^{w}-\left\langle n_{b}\right\rangle^{f} \geqq 1-O\left(q^{-1 / d}\right)$. Since the internal energy is given by $E(\beta)=-d f / d \beta=-e^{\beta}\left(e^{\beta}-1\right)^{-1}\left\langle n_{b}\right\rangle$, this shows the existence of a jump

$$
\Delta E=E\left(\beta_{t}-0\right)-E\left(\beta_{t}+0\right)>0,
$$

at $\beta_{t}$, and shows that the system undergoes a first order phase transition, with a latent heat $\Delta E$, when the inverse temperature $\beta$ is varied.

More generally, one can apply the results about cluster expansions of polymer systems (the situation for large $q$ is quite similar to that of ferromagnetic Ising model at low temperature and the methods of $[14,15]$ may be used, see also [16]), to the contour models defined in the theorem. We get then, provided that $q$ is large enough, convergent expansions in powers of $q^{-1 / d}$, of the logarithm of the partition functions and the correlation function under free and wired boundary conditions. Moreover, we may also consider general boundary conditions (although they give rise to "long contours") because the partition functions in finite volumes (these volumes correspond to the different regions separated by the "long contours" and present a free or a wired boundary) can also be written as a convergent expansion. These expansions, together with known arguments from the low temperature analysis of lattice systems $[14,17]$, allow us to recover (and extend to non-integer values of $q$ ) the following results of [4-8] and [18].

If $\beta \neq \beta_{t}$, there is only one translation invariant Gibbs state. It describes, for $\beta>\beta_{t}$, the ordered phase and for $\beta<\beta_{t}$, the disordered phase (for $q$ going to infinity these states approach the wired ground state if $\beta>\beta_{t}$, i.e. the Dirac delta measure whose support is the wired ground configuration, and the free ground state if $\beta<\beta_{t}$ ). At $\beta=\beta_{t}$ there are two pure phases, the ordered $\langle\cdot\rangle^{w}$ and the disordered $\langle\cdot\rangle^{f}$ states. Every translation invariant Gibbs state is then a convex linear combination of these two extremal states. Moreover, the truncated correlation functions associated to the pure phases decay exponentially and there is a strictly positive surface tension at $\beta_{t}$ between the ordered and the disordered phases.

Finally, let us point out that we are lead to introduce also mixed wired boundary 
conditions, which, in the usual version of the Potts model, correspond to fix the spin value $1,2, \ldots$ or $q$, at each site on the boundary. This may be done by restricting the allowed configurations of bonds to those which do not connect some given subsets of the boundary. Notice that these subsets can be given arbitrary, in a number independent of the value of $q$. On the other hand, by adding these non-connectedness conditions to the usual boundary conditions, we may extend the notion of Gibbs states and introduce a surface tension between two equivalent but distinct ordered phases. The above results concerning the number of pure phases in equilibrium remain true also for this extended notion of Gibbs states. Moreover for all $\beta \geqq \beta_{t}$ the ordered-ordered surface tension is strictly positive.

\section{Appendix}

In this appendix we prove the theorem stated in Sect. 2. We use the inductive approach of [19] and closely follow Sect. 2.5 of [7] to which we refer the reader for more details.

First Step. We shall construct the contour functionals $\phi_{w}$ and $\phi_{f}$ following the inductive procedure of [19]. First of all one observes that for every $\alpha_{w}, \alpha_{f} \geqq 0$ one may define, by induction in $\left|V\left(\gamma_{w}\right)\right|$ and $\left|V\left(\gamma_{f}\right)\right|$, the contour functionals $\phi_{w}^{\alpha_{w}}, \phi_{f}^{\alpha_{f}}$ using the inductive relations of Theorem 1 with $b_{w}=\alpha_{w}$ and $b_{f}=\alpha_{f}$. Let us artificially transform these functionals into $\tau$-functionals by defining

Define further

$$
\begin{gathered}
\bar{\phi}_{w}^{\alpha_{w}}(\gamma)=\min \left(\phi_{w}^{\alpha_{w}}(\gamma), e^{-\tau_{w}\|\gamma\|}\right), \\
\bar{\phi}_{f}^{\alpha_{f}}(\gamma)=\min \left(\phi_{f}^{\alpha_{f}}(\gamma), e^{-\tau_{f}\|\gamma\|}\right) .
\end{gathered}
$$

$$
\begin{aligned}
& b_{w}=\sup \left\{\alpha_{w} \mid \alpha_{w}+\ln \left(e^{\beta}-1\right)+f\left(\bar{\phi}_{w}^{\alpha_{w}}\right) \leqq f(\beta)\right\}, \\
& b_{f}=\sup \left\{\alpha_{f} \mid \alpha_{f}+\frac{1}{d} \ln q+f\left(\bar{\phi}_{f}^{\alpha_{f}}\right) \leqq f(\beta)\right\}
\end{aligned}
$$

We show that

$$
b_{w}+\ln \left(e^{\beta}-1\right)+f\left(\bar{\phi}_{w}^{b_{w}}\right)=b_{f}+\frac{1}{d} \ln q+f\left(\bar{\phi}_{f}^{b_{f}}\right)=f(\beta),
$$

by proving that $f\left(\bar{\phi}_{w}^{\alpha_{w}}\right)$ and $f\left(\bar{\phi}_{f}^{\alpha_{f}}\right)$ are continuous in $\alpha_{w}$ and $\alpha_{f}$. This follows from the fact that $(1 /|V|) \log \mathscr{Z}\left(V \mid \bar{\phi}^{\alpha}\right)$ is Lipschitz continuous uniformly in $V$ as may be seen by computing and bounding its derivative with respect to $\alpha$ (see [17]).

Second Step. We shall show by induction in $\left|V\left(\gamma_{w}\right)\right|$ and $\left|V\left(\gamma_{f}\right)\right|$ that $\phi_{w}^{b_{w}}$ and $\phi_{f}^{b_{f}}$ are actually $\tau$-functionals, i.e., that $\bar{\phi}_{w}^{b_{w}}=\phi_{w}^{b_{w}}$ and $\bar{\phi}_{f}^{b_{f}}=\phi_{f}^{b_{f}}$. Suppose that this is known for all $\hat{\gamma}_{w}$ with $\left|V\left(\hat{\gamma}_{w}\right)\right| \leqq k$ and all $\hat{\gamma}_{f}$ with $\left|V\left(\hat{\gamma}_{f}\right)\right| \leqq k+1$ and consider a contour $\gamma_{w}$ with $\left|V\left(\gamma_{w}\right)\right| \leqq k+1$. Notice first that from the induction hypothesis

$$
\left.\mathscr{Z}\left(\operatorname{Int} \gamma_{w} \mid \phi_{w}^{b_{w}}\right)=\mathscr{Z}\left(\operatorname{Int} \gamma_{w} \mid \bar{\phi}_{w}^{b_{w}}\right)\right)=\exp \left(f\left(\bar{\phi}_{w}^{b_{w}}\right)\left|\operatorname{Int} \gamma_{w}\right|+\sigma\left(\operatorname{Int} \gamma_{w} \mid \bar{\phi}_{w}^{b_{w}}\right)\right) \text {, }
$$

where

$$
\sigma(V \mid \phi)=\ln \mathscr{Z}(V \mid \phi)-|V| f(\phi) .
$$


Observing that for any contour $\hat{\gamma}_{f}$ contributing to $\mathscr{Z}\left(V\left(\gamma_{w}\right) \mid \bar{\phi}_{f}^{b}\right)$ one has $\hat{\gamma}_{f} \subset V\left(\gamma_{w}\right)$, one gets, using again the induction hypothesis,

$$
\mathscr{Z}\left(V\left(\gamma_{w}\right) \mid \phi_{f}^{b_{f}}\right)=\mathscr{Z}\left(V\left(\gamma_{w}\right) \mid \bar{\phi}_{f}^{b_{f}}\right)=\exp \left(f\left(\bar{\phi}_{f}^{b_{f}}\right)\left|V\left(\gamma_{w}\right)\right|+\sigma\left(V\left(\gamma_{w}\right) \mid \bar{\phi}_{w}^{b_{w}}\right)\right) .
$$

Starting from the definition, referring to (2.13), to the first step, to the above observations and to the obvious inequality

we get

$$
\mathscr{Z}\left(V\left(\gamma_{w}\right) \mid \phi_{f}^{b_{f}} ; b_{f}\right) \leqq \exp \left(b_{f}\left|V\left(\gamma_{w}\right)\right|\right) \mathscr{Z}\left(V\left(\gamma_{w}\right) \mid \phi_{f}^{b_{f}}\right),
$$

$$
\begin{aligned}
\phi_{w}^{b_{w}}\left(\gamma_{w}\right)= & e^{-b_{w}\left|V\left(\gamma_{w}\right)\right|}\left(e^{\beta}-1\right)^{-\left|V\left(\gamma_{w}\right)\right|} \frac{Z^{f}\left(V\left(\gamma_{w}\right)\right)}{\mathscr{Z}\left(\operatorname{Int} \gamma_{w} \mid \phi_{w}^{b_{w}}\right)} \\
= & e^{-b_{w}\left|V\left(\gamma_{w}\right)\right|}\left(e^{\beta}-1\right)^{-\left|V\left(\gamma_{w}\right)\right|} q^{\left|S_{I}\left(V\left(\gamma_{w}\right)\right)\right|} \frac{\mathscr{Z}\left(V\left(\gamma_{w}\right) \mid \phi_{f}^{b_{f}} ; b_{f}\right)}{\mathscr{Z}\left(\operatorname{Int} \gamma_{w} \mid \phi_{w}^{b_{w}}\right)} \\
\leqq & \exp \left\{\left|V\left(\gamma_{w}\right)\right|\left[-b_{w}-\ln \left(e^{\beta}-1\right)-f\left(\bar{\phi}_{w}^{b_{w}}\right)+b_{f}+f\left(\bar{\phi}_{f}^{b_{f}}\right)\right]\right\} \\
& \cdot \exp \left\{f\left(\bar{\phi}_{w}^{b_{w}}\right)\left|\gamma_{w}\right|+\sigma\left[V\left(\gamma_{w}\right) \mid \bar{\phi}_{f}^{b_{f}}\right]-\sigma\left[\operatorname{Int} \gamma_{w} \mid \bar{\phi}_{w}^{b_{w}}\right]\right\} q^{S\left(\operatorname{Int} \gamma_{w}\right)} \\
= & q^{\left|S\left(\operatorname{lnt} \gamma_{w}\right)\right|-1 / \alpha\left|\operatorname{Int} \gamma_{w}\right|-1 / d\left|\gamma_{w}\right|} \\
& \cdot \exp \left\{f\left(\bar{\phi}_{w}^{b_{w}}\right)\left|\gamma_{w}\right|+\sigma\left[V\left(\gamma_{w}\right) \mid \bar{\phi}_{f}^{b_{f}}\right]-\sigma\left[\operatorname{Int} \gamma_{w} \mid \bar{\phi}_{w}^{b_{w}}\right]\right\} .
\end{aligned}
$$

Then, by taking into account the lemma, we use

$$
\left|S\left(\operatorname{Int} \gamma_{w}\right)\right|-1 / d\left|\operatorname{Int} \gamma_{w}\right|-1 / d\left|\gamma_{w}\right|=-\left|S\left(\operatorname{Ext} \gamma_{w}\right)\right|+1 / d\left|\operatorname{Ext} \gamma_{w}\right|=-\frac{1}{2 d}\left\|\gamma_{w}\right\|,
$$

to get

$$
\phi_{w}\left(\gamma_{w}\right) \leqq q^{-(1 / 2 d)\left\|\gamma_{w}\right\|} \exp \left\{f\left(\bar{\phi}_{w}^{b_{w}}\right)\left|\gamma_{w}\right|+\sigma\left[V\left(\gamma_{w}\right) \mid \bar{\phi}_{f}^{b_{f}}\right]-\sigma\left[\operatorname{Int} \gamma_{w} \mid \bar{\phi}_{w}^{b_{w}}\right]\right\} .
$$

Similarly for $\gamma_{f}$ with $\left|V\left(\gamma_{f}\right)\right| \leqq k+2$, from the induction hypothesis we have

$$
\begin{aligned}
& \left.\mathscr{Z}\left(\operatorname{Int} \gamma_{f} \mid \phi_{f}^{b_{f}}\right)=\mathscr{Z}\left(\operatorname{Int} \gamma_{f} \mid \bar{\phi}_{f}^{b_{f}}\right)\right)=\exp \left(f\left(\bar{\phi}_{f}^{b_{f}}\right)\left|\operatorname{Int} \gamma_{f}\right|+\sigma\left(\operatorname{Int} \gamma_{f} \mid \bar{\phi}_{f}^{b_{f}}\right)\right), \\
& \left.\mathscr{Z}\left(\operatorname{Int} \gamma_{f} \mid \phi_{w}^{b_{w}}\right)=\mathscr{Z}\left(\operatorname{Int} \gamma_{f} \mid \bar{\phi}_{w}^{b_{w}}\right)\right)=\exp \left(f\left(\bar{\phi}_{w}^{b_{w}}\right)\left|\operatorname{Int} \gamma_{f}\right|+\sigma\left(\operatorname{Int} \gamma_{f} \mid \bar{\phi}_{w}^{b_{w}}\right)\right),
\end{aligned}
$$

so that

$$
\begin{aligned}
\phi_{f}^{b_{f}}\left(\gamma_{f}\right) & =e^{-b_{f}\left|V\left(\gamma_{f}\right)\right|} q^{-\left|S\left(\operatorname{Int} \gamma_{f}\right)\right|} \frac{Z^{w}\left(\operatorname{Int} \gamma_{f}\right)}{\mathscr{Z}\left(\operatorname{Int} \gamma_{f} \mid \phi_{f}^{b_{f}}\right)} \\
& \leqq q^{-\left\{\left|\boldsymbol{S}\left(\operatorname{Int} \gamma_{f}\right)\right|-(1 / d)\left|\operatorname{Int} \gamma_{f}\right|+C\left(\operatorname{Int} \gamma_{f}\right)\right\}} \exp \left\{\sigma\left[\operatorname{Int} \gamma_{f} \mid \bar{\phi}_{w}^{b_{w}}\right]-\sigma\left[\operatorname{Int} \gamma_{f} \mid \bar{\phi}_{f}^{b_{f}}\right]\right\} \\
& \leqq q^{-(1 / 2 d)\left\|\gamma_{f}\right\|+C\left(\operatorname{Int} \gamma_{f}\right)} \exp \left\{\sigma\left[\operatorname{Int} \gamma_{f} \mid \bar{\phi}_{w}^{b_{w}}\right]-\sigma\left[\operatorname{Int} \gamma_{f} \mid \bar{\phi}_{f}^{b_{f}}\right]\right\} \\
& \leqq q^{-[(d-1) /(4 d-2) d]\left\|\gamma_{f}\right\|} \exp \left\{\sigma\left[\operatorname{Int} \gamma_{f} \mid \bar{\phi}_{w}^{b_{w}}\right]-\sigma\left[\operatorname{Int} \gamma_{f} \mid \bar{\phi}_{f}^{b_{f}}\right]\right\},
\end{aligned}
$$

where we used $\mathbf{C}\left(\operatorname{Int} \gamma_{f}\right) \leqq[1 /(4 d-2)]\left\|\gamma_{f}\right\|$ in the last inequality.

We take $\tau_{w}=(1 / 2 d) \ln q-\ln K$ and $\tau_{f}=[(d-1) /(4 d-2) d] \ln q-\ln K$, evaluate $\left|f\left(\bar{\phi}_{w}^{b_{w}}\right)\right| \leqq e^{-\tau_{w}}$, use that (cf. [11]): $\left|\sigma\left(V \mid \bar{\phi}_{w}^{b_{w}}\right)\right| \leqq|\partial V| e^{-\tau_{w}}$ and $\left|\sigma\left(V \mid \bar{\phi}_{f}^{b_{f}}\right)\right| \leqq|\partial V| e^{-\tau_{f}}$, to get the estimates of the theorem (obtained with $K=3$ ) and also the equality of free energies stated there, which actually is identical with that stated in the first step since $\bar{\phi}_{w}^{b_{w}}=\phi_{w}^{b_{w}}$ and $\bar{\phi}_{f}^{b_{f}}=\phi_{f}^{b_{f}}$ for $q^{-[(d-1) /(4 d-2) d]} \geqq(3 K / \ln K)$. 
Third Step. Next we prove that $\min \left(b_{w}, b_{f}\right)=0$. If we suppose that $\min \left(b_{w}, b_{f}\right)$ $>0$ then, referring to the fact that the Lipschitz constants of $f\left(\bar{\phi}_{w}^{b_{w}}\right)$ and $f\left(\bar{\phi}_{f}^{b_{f}}\right)$ are at most $1 / 2$, one would prove that there exists $\varepsilon>0$ and parameters $\tilde{b}_{w}, \tilde{b}_{f}$ such that

$$
\tilde{b}_{w}+\ln \left(e^{\beta}-1\right)+f\left(\bar{\phi}_{w}^{\tilde{b}_{w}}\right)=\tilde{b}_{f}+\frac{1}{d} \ln q+f\left(\bar{\phi}_{f}^{\tilde{b}_{f}}\right)=f(\beta)-\varepsilon
$$

Using this equality of free energies instead of that stated in the theorem (where $\varepsilon=0$ ) one might show in the same way as above that $\bar{\phi}_{w}^{\tilde{b}_{w}}$ and $\bar{\phi}_{f}^{\tilde{b}_{f}}$ are $\tau$-functionals and thus

$$
\tilde{b}_{w}+\ln \left(e^{\beta}-1\right)+f\left(\bar{\phi}_{w}^{\tilde{b}_{w}}\right)=\tilde{b}_{f}+\frac{1}{d} \ln q+f\left(\bar{\phi}_{f}^{\tilde{b}_{f}}\right)<f(\beta)
$$

in contradiction with

$$
f(\beta) \leqq \tilde{b}_{w}+\ln \left(e^{\beta}-1\right)+f\left(\bar{\phi}_{w}^{\tilde{b}_{w}}\right)
$$

which follows from

$$
Z^{w}(V)=q\left(e^{\beta}-1\right)^{|V|} \mathscr{Z}\left(V \mid \phi_{w}^{\tilde{b}_{w}} ; \tilde{b}_{w}\right) \leqq q\left(e^{\beta}-1\right)^{|V|} e^{\tilde{b}_{w}|V|} \mathscr{Z}\left(V \mid \phi_{w}^{\tilde{b}_{w}}\right) .
$$

Therefore $\min \left(b_{w}, b_{f}\right)=0$.

Fourth Step. Finally we prove that for a fixed $q$, there exists a unique $\beta_{t}$ for which $b_{w}=b_{f}=0$. We notice that at such a point necessarily $\ln \left(e^{\beta}-1\right)+f\left(\phi_{w}^{0}\right)=$ $(1 / d) \ln q+f\left(\phi_{f}^{0}\right)$. Thus our aim is to prove that the two corresponding curves intersect in a single point. At the point $\beta_{0}$, solution of the equation $e^{\beta}-1=q^{1 / d}$, the slope of the tangent to the graph of $\ln \left(e^{\beta}-1\right)$ is $1+q^{-1 / d}$, and differs significantly from zero, the slope of the line $(1 / d) \ln q$. Taking into account that $\left|f\left(\phi_{w}^{0}\right)\right|$ and $\left|f\left(\phi_{f}^{0}\right)\right|$ as well as $\left|(d / d \beta) f\left(\phi_{w}^{0}\right)\right|$ and $\left|(d / d \beta) f\left(\phi_{f}^{0}\right)\right|$ may be bounded by $e^{-\tau}$, one shows that $\ln \left(e^{\beta}-1\right)+f\left(\phi_{w}^{0}\right)$ and $(1 / d) \ln q+f\left(\phi_{f}^{0}\right)$ are respectively contained in a tiny strip around $\ln \left(e^{\beta}-1\right)$ and $(1 / d) \ln q$, and have entirely different slopes. Thus one may conclude that they intersect in a single point $\beta_{t}$ near $\beta_{0}$. The bounds on $\left|(d / d \beta) f\left(\phi_{w}^{0}\right)\right|$ and $\left|(d / d \beta) f\left(\phi_{f}^{0}\right)\right|$ are shown as in the proof of the Lipshitz continuity in the first step. We again use the inductive relation with $\alpha_{w}=\alpha_{f}=0$ and evaluate $\left|(d / d \beta) \ln Z\left(V(\gamma) \mid \phi_{w}^{0}\right)\right|$ and $\left|(d / d \beta) \ln Z\left(\operatorname{Int} \gamma \mid \phi_{f}^{0}\right)\right|$ in terms of the mean energies in the ensembles corresponding to the wired and the free partition functions. This ends the proof of the theorem.

Acknowledgement. L. L. and S. S. are grateful to C.P.T. for warm hospitality and financial support.

\section{References}

1. Potts, R. B.: Some generalized order-disorder transformation. Proc. Camb. Phil. Soc. 48, 106 (1952)

2. Wu, F. Y.: The Potts model. Rev. Mod. Phys. 54, 235 (1982)

3. Baxter, R. J.: Potts model at the critical temperature. J. Phys. C6, L445 (1973); Magnetization discontinuity of the two-dimensional Potts model. J. Phys. A15, 3329 (1982)

4. Kotecky, R., Shlosman, S. B.: First order phase transitions in large entropy lattice systems. Commun. Math. Phys. 83, 493 (1982) 
5. Bricmont, J., Kuroda, K., Lebowitz, J. L.: First order phase transitions in lattice and continuous systems. Commun. Math. Phys. 101, 501 (1985)

6. Laanait, L., Messager, A., Ruiz, J.: Phase coexistence and surface tensions for the Potts model. Commun. Math. Phys. 105, 527 (1986)

7. Kotecky, R., Laanait, L., Messager, A., Ruiz, J.: The $q$-state Potts model in the standard Pirogov-Sinaï theory: Surface tensions and Wilson loops. J. Stat. Phys. 58, 199 (1990)

8. Dinaburg, E. I., Sinaï, Ya. G.: Contour models with interactions and their applications. Selecta Math. Sov. vol. 7, (3) (1988)

9. Fortuin, C. M., Kasteleyn, P. W.: On the random cluster model. Physica 57, 536 (1972)

10. Pirogov, S. A., Sinaï, Ya. G.: Phase diagrams of classical lattice systems. Theor. Math. Phys. 25, 1185 (1975), and Theor. Math. Phys. 26, 39 (1976)

11. Sinaï, Ya. G.: Theory of Phase Transitions: Rigorous Results. London: Pergamon Press 1982

12. Aizenman, M., Chayes, J. T., Chayes, L., Newman, C. M.: Discontinuity of the magnetization in one-dimensional $1 /|x-y|^{2}$ Ising and Potts models. J. Stat. Phys. 50, 1 (1988)

13. Aizenman, M., Chayes, J. T., Chayes, L., Newman, C. M.: The phase boundary in dilute and random Ising and Potts ferromagnets. J. Phys. A20, L313 (1987)

14. Gallavotti, G., Martin-Löf, A., Miracle-Solé, S.: Some problems connected with the description of coexisting phases at low temperature in the Ising model. In: Lecture Notes in Physics, Vol. 20, Berlin, Heidelberg, New York: Springer 1973

15. Seiler, E.: Gauge theories as a problem of constructive quantum field theory and statistical mechanics. Lecture Notes in Physics, Vol. 159, Berlin, Heidelberg, New York: Springer 1982

16. Kotecky, R., Preiss, D.: Cluster expansion for abstract polymer models. Commun. Math. Phys. 103, 491 (1986)

17. Zahradnik, M.: An alternate version of Pirogov-Sinaï theory. Commun. Math. Phys. 93, 559 (1984)

18. Martirosian, D. H.: Translation invariant Gibbs states in the $q$-state Potts model. Commun. Math. Phys. 105, 281 (1986)

19. Kotecky, R., Preiss, D.: An inductive approach to Pirogov-Sinaï theory. Rend. Circ. Matem. Palermo, II(3), 161 (1984)

Communicated by Ya. G. Sinaï 
